\title{
Physical and Chemical Aspects of the Nucleation of Cement-Based Materials
}

\author{
Pavel Demo ${ }^{1,2}$, Alexey Sveshnikov ${ }^{1,2}$, Šárka Hošková ${ }^{1}$, David Ladman ${ }^{1}$, Petra Tichá1 \\ ${ }^{1}$ Institute of Physics, Academy of Sciences of the Czech Republic, Cukrovarnická 10, 16253 Praha 6, Czech Republic \\ ${ }^{2}$ Czech Technical University in Prague, Faculty of Civil Engineering, Thákurova 7, 16629 Praha 6, Czech Republic
}

Corresponding author: demo@fzu.cz

\begin{abstract}
A theoretical model of the nucleation of portlandite is proposed, and the critical size of a portlandite cluster and the energy barrier of nucleation are determined. The steady state nucleation rate and the time lag of the nucleation of portlandite are estimated for a pure solution of $\mathrm{Ca}(\mathrm{OH})_{2}$ in water. Possible connections with the corresponding properties for cement paste are discussed. A new method is developed for experimentally determining the concentration of $\mathrm{Ca}^{2+}$ ions during the initial stage of hydration of a cement paste. The time dependence of $\mathrm{Ca}^{2+}$ ions is measured for various water-to-cement ratio values. The results are discussed from the point of view of existing models of the induction period.
\end{abstract}

Keywords: cement paste, induction period, calcium concentration, nucleation rate, time lag.

\section{Introduction}

Cementitious materials are one of the most widelyused types of materials in modern civil engineering. Concrete has many advantages, such as very high compressive strength, good resistance to corrosion and fire, and good acoustic damping. Workability occupies a special place among these properties of concrete. Workability refers to the ability of a fresh cement paste to fill the desired shape without reducing the quality of the concrete. High workability of cement paste over a period of several hours significantly reduces construction costs, and enables both easier shaping of the material and pre-casting of concrete that can then be delivered to the construction site. Having in mind the great importance of the workability of cement paste, extensive research has been carried out in order to understand the underlying mechanism and to study the possibility of extending the workability period without a significant impact on the properties of the final material. Despite the efforts that have been made, there are still many unanswered questions due to the very complex nature of the physico-chemical transformations taking place in the hydrating cement paste.

The hydration process of cement paste can be divided into a number of main stages. The first stage is the initial wetting of the cement particles and rapid dissolution of a small amount of the cement minerals, especially the calcium aluminates and the alkali sulfates. This stage is accompanied by significant heat production and lasts only few minutes. During this stage the hydrating tricalcium silicate also releases a large number of calcium ions into the solution, due to the very high intrinsic solubility of calcium. After the initial stage of exothermic reactions, the cement paste "falls asleep", and for the next few hours practically no changes are observed in the state of the paste. The duration of this so-called dormant (or induction) period directly determines the workability, since the strength of the paste begins to grow steadily after the dormant period finishes and the concrete begins to set. Thus, it is important to understand the reasons for the existence of the induction period and possible ways to influence its duration without a negative outcome.

Several hypotheses have been proposed in order to explain the induction period. Taylor has grouped them into four main classes [9]:

1. The initial nuclei of $\mathrm{C}-\mathrm{S}-\mathrm{H}$ and $\mathrm{CH}$ are somehow poisoned by $\mathrm{SiO}_{2}$ and cannot grow until the solution becomes supersaturated [8].

2. The membrane hypothesis assumes, that during the first stage of paste hydration a semipermeable membrane forms around the particles, thus separating the solution into an inner part and an outer part. The membrane prevents the ions dissolved in the inner solution from reaching the main volume of the solution. According to this hypothesis, the induction period ends when the osmotic pressure inside the membrane grows high enough to break it $[1,6]$.

3. The protective layer hypothesis is similar to the membrane hypothesis, but supposes that the 


\begin{tabular}{lccccccccc}
\hline Oxide & $\mathrm{CaO}$ & $\mathrm{SiO}_{2}$ & $\mathrm{Al}_{2} \mathrm{O}_{3}$ & $\mathrm{Fe}_{2} \mathrm{O}_{3}$ & $\mathrm{MgO}$ & $\mathrm{SO}_{3}$ & $\mathrm{Na}_{2} \mathrm{O}$ & $\mathrm{K}_{2} \mathrm{O}$ & $\mathrm{MnO}$ \\
\hline Comp. [wt.\%] & 63.77 & 20.51 & 4.74 & 3.3 & 1.05 & 3.07 & 0.15 & 0.95 & 0.09 \\
\hline
\end{tabular}

Table 1: Oxide composition of CEM I 42.5R, Mokrá, Czech Republic

\begin{tabular}{lcccccc}
\hline Mineral & $\mathrm{C}_{3} \mathrm{~S}$ & $\mathrm{C}_{2} \mathrm{~S}$ & $\mathrm{C}_{3} \mathrm{~A}$ & $\mathrm{C}_{4} \mathrm{AF}$ & $\mathrm{C}_{2} \mathrm{~F}$ & $\mathrm{SO}_{3}$-bound $\mathrm{CaO}$ \\
\hline Comp. [wt.\%] & 58.34 & 14.8 & 6.81 & 10.31 & 0 & 2.149 \\
\hline
\end{tabular}

Table 2: Phase composition of CEM I 42.5R, Mokrá, Czech Republic (Bogue)

semipermeable layer is formed directly on the surface of the grains. As in the case of the membrane hypothesis, the induction period ends when the protective layer is somehow removed or made permeable.

4. The nucleation hypothesis attributes the existence of the induction period to the very mechanism of the formation and growth of the nuclei. The growing particles of the new phase have to overcome a certain energetical barrier by means of fluctuations, and this cannot happen instantly. This hypothesis explains the induction period as an intrinsic time lag of the nucleation process $[3,10]$.

In our paper we analyze a nucleation hypothesis of the induction period. The hydrating cement paste is an extremely complex system. For our analysis, we concentrated our attention on one of the main phases in the hydrating paste - portlandite $\mathrm{Ca}(\mathrm{OH})_{2}$. We measured the concentration of $\mathrm{Ca}^{2+}$ in a hydrating cement paste, and combined this with theoretical modelling of the nucleation of a water solution of calcium hydroxide to estimate the nucleation time lag of portlandite formation.

\section{Experimental}

Materials. In our experiments we used an ordinary Portland cement (OPC) CEM I 42.5R, Mokrá, Czech Republic. The oxide and mineralogical composition of the cement is given in Tables 1 and 2 .

Chemicals. All chemical agents applied are of purity p.a. 0.05 M EDTA, disodium ethylenediaminetetraacetate, $0.05 \mathrm{M} \mathrm{MgSO}_{4}$, Schwarzenbach buffer, Eriochrome black T.

Method. The concentration of $\mathrm{Ca}^{2+}$ ions at an early stage of hydration of the cement paste was measured. The method for determining free calcium ions [5] is essentially based on the use of a chelatometric titration method, namely determining the concentration of $\mathrm{Ca}^{2+}$ ions by disodium ethylenediaminetetraacetate (EDTA, Chelaton III), a basic volumetric (titration) method in analytical chemistry. It is well known that chelatometric determination utilizes the fact that EDTA forms very strong compounds (chelates) with certain metals belonging to the II, III and IV group. Calcium is one of these metals. However, in our method for determining calcium ions in the first two hours of setting of the cement paste, EDTA plays a more complex role.

During the first two hours after the cement is mixed with water, the reactions run very quickly and the release of $\mathrm{Ca}^{2+}$ can be very rapid. The reaction is exothermic, as shown by the calorimetric curves (see Figure 1).

In order to measure the time dependence of the concentration of the calcium ions in the hydrating cement pastes, it is neccessary to stop the reaction at a chosen instant. The hydration reaction was stopped with the use of EDTA, which is simultaneously a strong retardant for the reaction of cement with water. Thus this acid serves both as the retardant and as the volumetric reagent for calcium ions. To improve the accuracy of the method, we used indirect titration, i. e. the amount of excess EDTA was determined by reaction with magnesium ions, and the $\mathrm{Ca}^{2+}$ concentration was calculated from the difference.

The concentration of $\mathrm{Ca}^{2+}$ ions as a function of time was determined for various water-to-cement ratios $(w / c=0.35 ; 0.40 ; 0.45 ; 0.50 ; 0.60)$, and the results are presented in Figure 2. All the curves have a peak at the very beginning of the hydration process, which corresponds to the first stage of intense exothermic reactions. Afterwards the concentration drops. This decrease in free ion concentration can be explained as the formation of some protective layer or membrane. When the osmotic pressure exceeds a certain threshold, the membrane bursts and releases the $\mathrm{Ca}^{2+}$ ions held within, leading to a later increase in concentration. At this instant, the nucleation mechanism kicks in, and portlandite nuclei begin to form. From the point of view of nucleation 


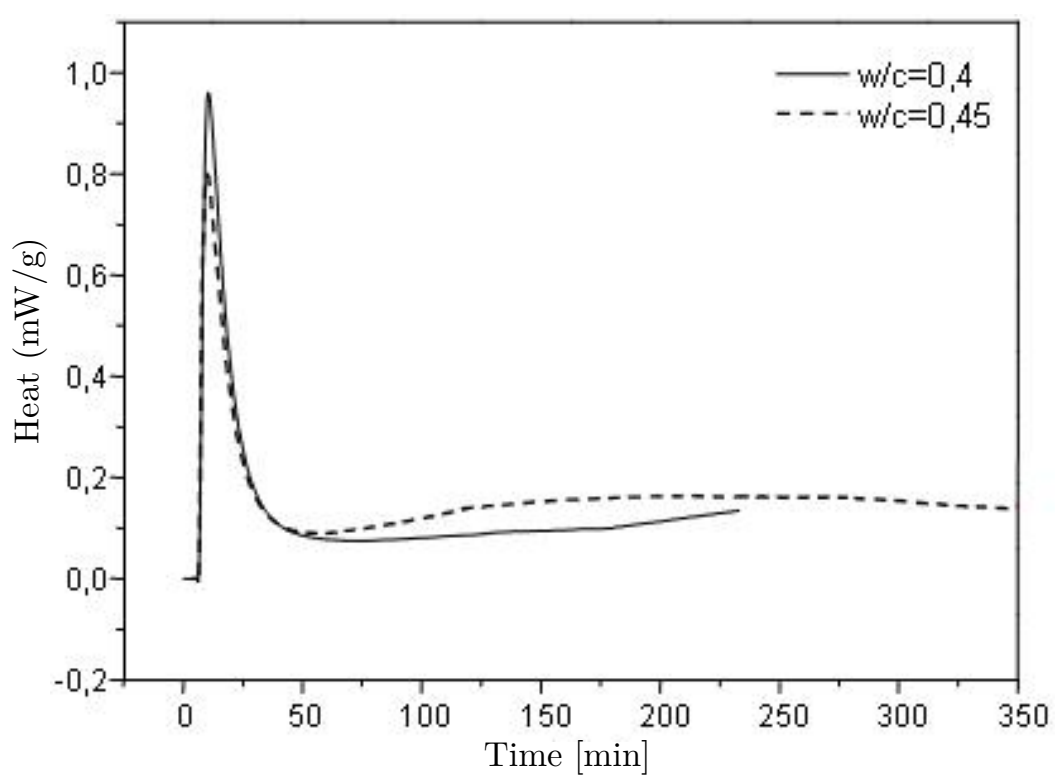

Figure 1: Initial stage of the hydration process in HCP of Portland cement, Mokrá, $(w / c=0.40 ; 0.45)$ measured using a SETARAM C 80 calorimeter.

theory, the $\mathrm{Ca}^{2+}$ ions serve as monomers for future portlandite clusters. These monomers are absorbed in the nucleation process, leading to a new decrease in calcium ion concentration and the establishment of the second peak on the curves in Figure 2.

\section{Model}

According to nucleation theory, the initial stage of the process of the first order phase transition consists of two steps - nucleation and growth. Before the first particles of the new, stable phase can start to grow steadily, they have to appear somehow in the metastable phase, and this process is not easy for them. Although the resulting phase is more favorable from the energetical point of 1 view, the high surface-to-volume ratio of small particles combined with positive excess interface energy makes them unstable. Thus, nucleation theory distinguishes nucleation itself, when the embryos of the stable phase are stochastically produced in the volume of the metastable phase by means of fluctuations, and growth, when the nuclei increase in size deterministically. Depending on the system under consideration, the establishment of a steady growth rate can take a significant period of time, and this is called the time-lag of nucleation [11].

Portlandite clusters grow from a solution containing $\mathrm{Ca}^{2+}$ and $\mathrm{OH}^{-}$ions. We assume that hydroxyl ions are present in abundance, because they are not only a product of $\mathrm{Ca}(\mathrm{OH})_{2}$ dissociation, but also a product of dissociation of water itself. Thus, the formation of portlandite clusters is controlled by the supersaturation of calcium ions:

$$
S=\frac{C_{a c t}}{C_{e q}},
$$

where $C_{a c t}$ represents the actual concentration of $\mathrm{Ca}^{2+}$ ions within the solution and $C_{e q}$ is their equilibrium concentration. The Gibbs free energy of portlandite cluster formation is

$$
\Delta G=\Delta G_{V}+\Delta G_{S}
$$

where $\Delta G_{V}$ stands for the volume term, and $\Delta G_{S}$ is the surface term. The volume term is negative, because the solid phase as a whole is stable and energetically more favorable than the solution. Conversely, the surface term is positive and reflects the general tendency of a system to eliminate internal interfaces and become homogeneous.

The exact interplay between the volume and the surface terms in (2) depends on the shape of the growing cluster. It has been observed [2] that small crystals of portlandite are shaped as hexagonal plates (see Figure 3). The fact that the radius of crystal $r$ is much larger than its height $h$ suggests that the surface tension in this case is strongly anisotropic. Denoting the surface tension on the corresponding planes as $\sigma_{r}$ and $\sigma_{h}$, we obtain

$$
\Delta G=-k T \frac{3 \sqrt{3} r^{2} h}{2 v} \ln S+3 \sqrt{3} r^{2} \sigma_{r}+6 r h \sigma_{h}
$$

where $k=1.38 \times 10^{-23} \mathrm{~J} / \mathrm{K}$ is the Boltzmann constant, $T$ stands for absolute temperature, and $v=$ 


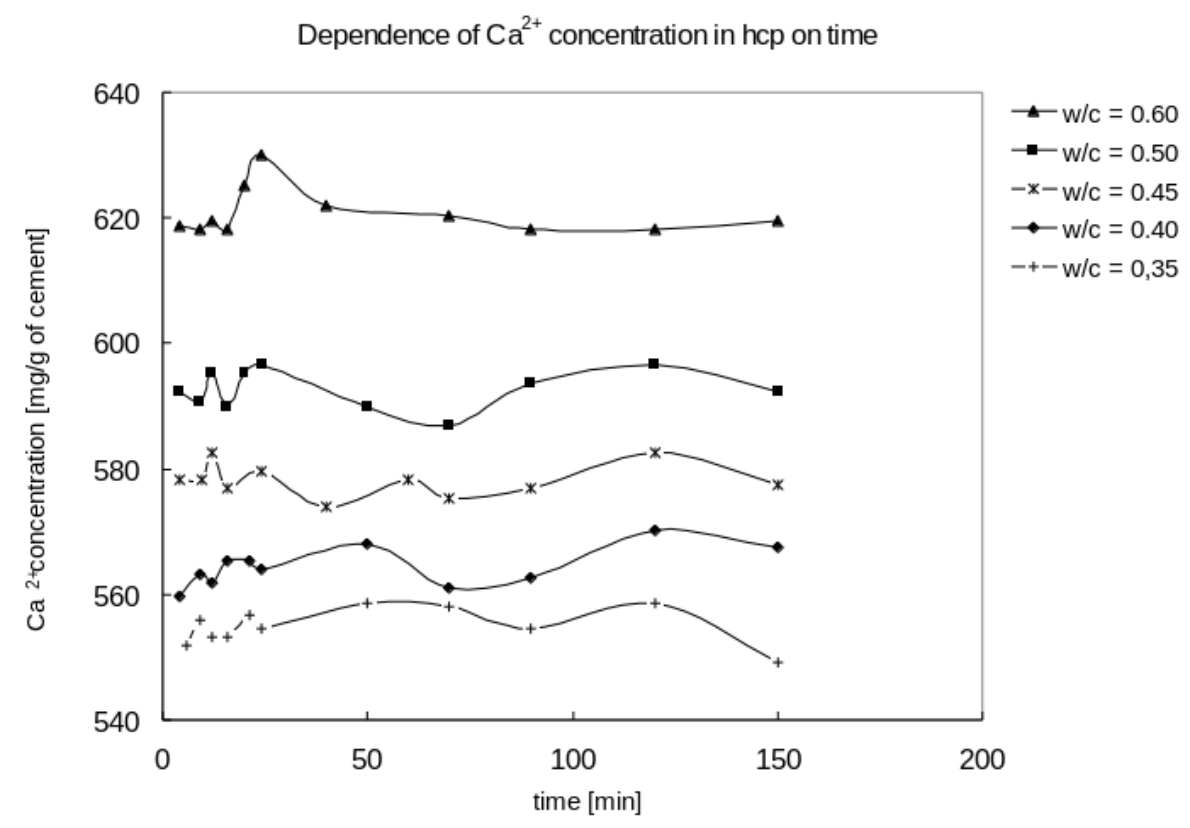

Figure 2: Concentration of $\mathrm{Ca}^{2+}$ ions in $\mathrm{HCP}$ vs. time related to the total mass of $\mathrm{HCP}\left(\mathrm{t}=25^{\circ} \mathrm{C}\right)$.

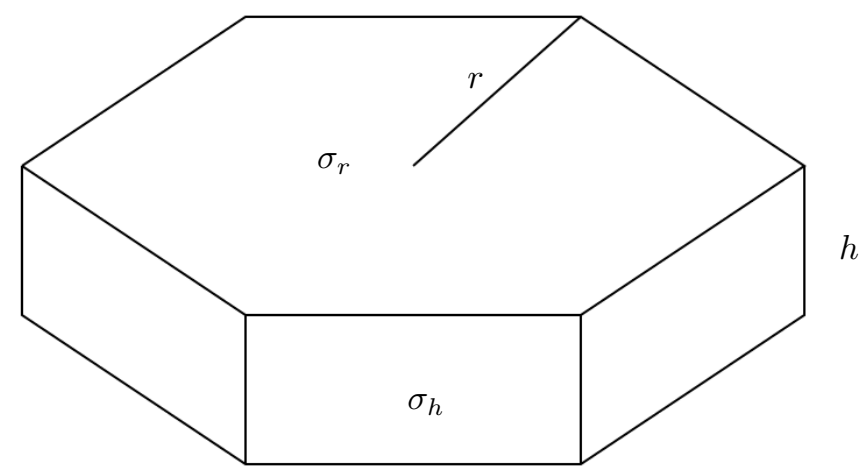

Figure 3: The assumed shape of a portlandite cluster and the corresponding surface energies.

$1.65 \times 10^{-28} \mathrm{~m}^{3}$ is the volume of a unit cell of portlandite, which is hexagonal with basis cell dimensions $a=0.3585 \mathrm{~nm}$ and $c=0.4895 \mathrm{~nm}$.

Under given supersaturation $S$, expression (3) is a function of two variables, $r$ and $h$. This expression describes the energetical landscape on which growing clusters have to find the optimal way to the valley, representing the stable solid phase. The optimal way leads through a saddle point of the energetical surface (3), and corresponds to the best thermodynamical approximation to the shape of a cluster. If we take kinetic aspects of cluster growth into account, it is possible that the dynamically changing shape of a growing crystal does not pass exactly through the saddle point of the energetical landscape. However, this deviation is negligible in most situations, so in the following text we assume that the shape of a critical cluster is determined from the pair of equations

$$
\left.\frac{\partial \Delta G}{\partial r}\right|_{r_{c}, h_{c}}=0,\left.\quad \frac{\partial \Delta G}{\partial h}\right|_{r_{c}, h_{c}}=0 .
$$

Solution of the system (4) yields

$$
r_{c}=\frac{4 \sigma_{h} v}{\sqrt{3} k T \ln S}, \quad h_{c}=\frac{4 \sigma_{r} v}{k T \ln S} .
$$

Clusters which are smaller than the critical cluster tend to dissolve, while larger clusters can grow freely. This last conclusion is valid for an open system, but in a closed system the situation is more complicated. In a closed system, the concentration of monomers is not constant, as they are consumed by the growing clusters. Thus, supersaturation $S$ is constantly decreasing, which leads to an increase in the critical sizes $r_{c}$ and $h_{c}$. It may even be possible that in later stages of the nucleation process, the 


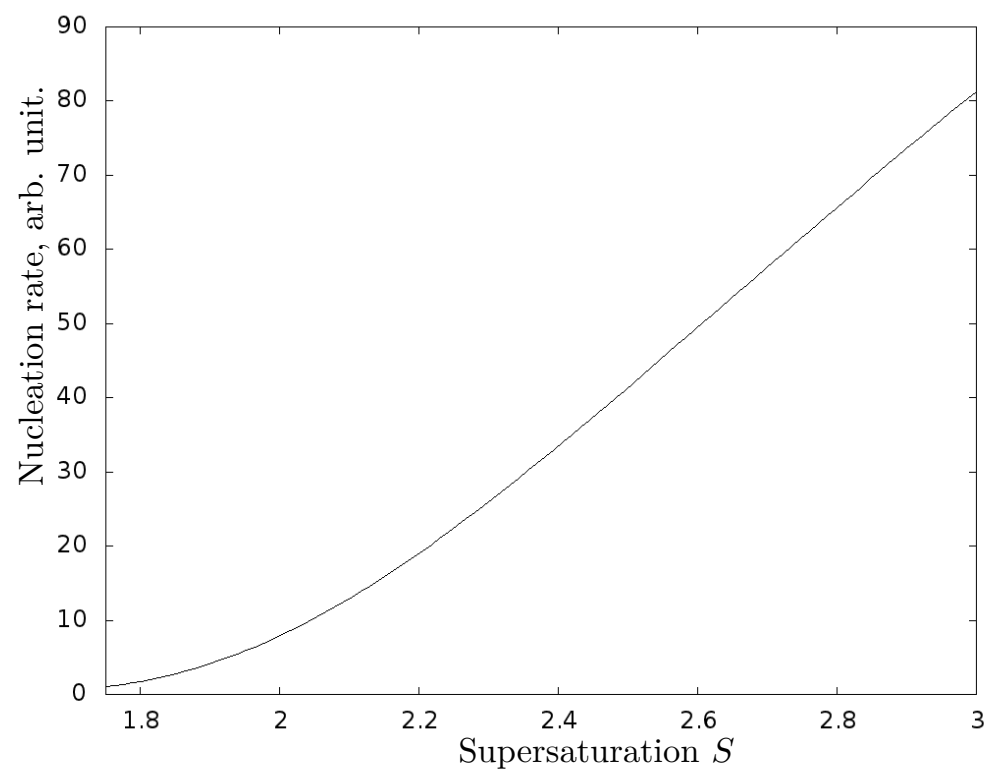

Figure 4: Dependence of the steady state nucleation rate of portlandite on the supersaturation of $\mathrm{Ca}^{2+}$ ions.

critical size grows faster than the front of the distribution function of the cluster. Then the clusters that have already originated and are overcritical may become undercritical again and dissolve. This effect of depletion of $\mathrm{Ca}^{2+}$ monomers due to their consumption by growing portlandite is shown in Figure 2 as a decrease in concentration after it achieves the second maximum. However, during the whole process the ratio of the radius of the critical platelet to its height remains constant and independent of supersaturation $S$ :

$$
\frac{r_{c}}{h_{c}}=\frac{\sigma_{h}}{\sqrt{3} \sigma_{r}} .
$$

Since $r_{c} \gg h_{c}$, lateral surface tension $\sigma_{h}$ must be much larger than basal $\sigma_{r}$. When a macroscopic measurement of the surface tension of portlandite is performed, it yields the surface energy value, averaged over the surface of the whole cluster. It can easily be shown from (6) that the contribution of the basal surface energy to the total energy of the cluster is four times smaller than the corresponding contribution of the lateral surface energy. Consequently, macroscopic measurements of the surface energy of portlandite provide values for $\sigma_{h}$.

After substituting (5) into (3), we obtain an expression for the energy barrier of nucleation:

$$
\Delta G=\frac{16 \sqrt{3} \sigma_{h}^{2} \sigma_{r} v^{2}}{(k T \ln S)^{2}} .
$$

If we take the values for calcium hydroxide supersaturation from [4], $S \in(1.75,3)$, then it is possible to obtain the following estimations for the critical size of a portlandite cluster at $T=298 \mathrm{~K}$ :

$$
r_{c} \in(5.1 \mathrm{~nm}, 10 \mathrm{~nm}), \quad h_{c} \in(1 \mathrm{~nm}, 2 \mathrm{~nm}) .
$$

The corresponding energy barrier

$$
\Delta G \in(7.2 \mathrm{eV}, 26.5 \mathrm{eV}) .
$$

However, these estimations were performed using the equilibrium concentration of $\mathrm{Ca}^{2+}$ ions based on the solubility curve of calcium hydroxide in water. Data for the calcium hydroxide solution in the cement paste is not available. We can only assume that the nucleation of portlandite is the dominant process during the induction period and, consequently, the equilibrium concentration of $\mathrm{Ca}^{2+}$ does not differ significantly from the pure solution. It is still an open question, though, how to relate the water-to-cement ratio of the cement paste to supersaturation of the solution with respect to portlandite.

Despite the relatively small variations in the critical size and the energy barrier, the steady state nucleation rate can be quite sensitive to supersaturation and, thus, to the water-to-cement ratio. This is explained by the fact that the nucleation rate (i. e. the number of new supercritical clusters appearing per unit volume of the system per unit time) is exponentially dependent on the energy barrier of nucleation:

$$
I \sim \exp \left(-\frac{\Delta G_{c}}{k T}\right) .
$$

A detailed knowledge of the kinetics of cluster growth is necessary for calculating the exact proportionality coefficient in (10). Nevertheless, relation (10) enables us to estimate the relative change in the nucleation rate under normal conditions (see Figure 4):

$$
\frac{I_{\min }}{I_{\max }} \simeq 80
$$




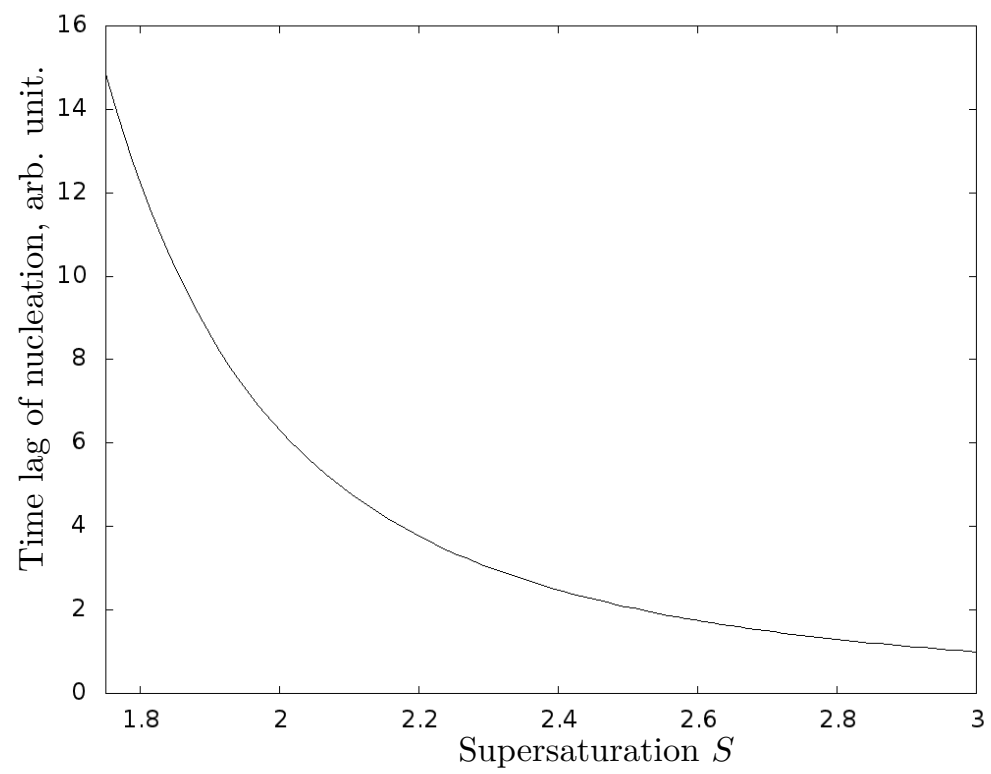

Figure 5: Time lag of the nucleation of portlandite in dependence on supersaturation of $\mathrm{Ca}^{2+}$ ions.

Equation (10) gives the nucleation rate in the steady state. However, as discussed above, due to the stochastic nature of the nucleation process, the nucleation rate does not achieve its steady state value immediately after the creation of supersaturation in the system. The corresponding time lag of nucleation can be estimated as [7]

$$
\tau=\frac{1}{2.8 Z^{2} R}
$$

where $Z$ is the so-called Zeldovich factor, which describes the curvature of the energetical landscape (3) at its saddle point, and $R$ stands for the rate at which monomers are incorporated into critical-size clusters. The Zeldovich factor $Z$ is equal to

$$
Z=\sqrt{\frac{-1}{2 \pi k T} \frac{\partial^{2} \Delta G_{c}}{\partial n^{2}}},
$$

where $n$ is the number of monomers inside the cluster. Figure 5 shows the dependence of the time lag of portlandite nucleation on supersaturation $S$.

\section{Conclusion}

We have proposed a new method for experimentally determining the concentration of $\mathrm{Ca}^{2+}$ ions in hydrating cement paste during the first few hours after mixing cement with water. The method is based on a standard chelatometric titration method, but uses EDTA both as the retardant of the hydration process and as the volumetric reagent for calcium ions. The evolution of calcium ion concentration has been studied bye this method for several $w / c$ ratios. The resulting curves (see Figure 2) express in general a bimodal behavior. The existence of the first maximum is attributed to the release of $\mathrm{Ca}^{2+}$ ions during the initial fast process of hydration of C-S-H, followed by the formation of some sort of protective layer around the cement grains. During the induction period, the concentration of calcium ions slowly grows due to the gradual hydration process. However, at later times the concentration goes down again because of the consumption of $\mathrm{Ca}^{2+}$ by nucleating and growing portlandite clusters.

Homogeneous nucleation theory is applied to portlandite cluster formation. We derive expressions for critical size and the energy barrier. Unfortunately, no data is available on the equilibrium concentration of $\mathrm{Ca}^{2+}$ ions in a cement paste. We estimate the critical size and the energy barrier of nucleation on the basis of the solubility curve of portlandite in water. Next, the expressions for the steady state nucleation rate and the time lag are obtained. The time lag of nucleation is strongly dependent on supersaturation (see Figure 5).

In the future, we plan to use our theoretical and experimental results to find the equilibrium concentration of $\mathrm{Ca}^{2+}$ ions in a cement paste, with a corresponding recalculation of the critical size and the energy barrier. If we assume that the major part of the induction period is due to the nucleation time lag, the comparison of the experimental data for the dependence of the duration of the induction period on the water-to-cement ratio, together with Figure 5, can provide information about the supersaturation of portlandite in a cement paste. In combination with data about the concentration of $\mathrm{Ca}^{2+}$ ions (Figure 2), it may be possible to calculate the solubility curve of calcium hydroxide in a cement paste. 


\section{Acknowledgements}

This study was supported by the Ministry of Education, Youth and Sport of the Czech Republic, project No. CEZ MSM 6840770003 and grant SGS10/125/OHK1/2T/11.

\section{References}

[1] P. Brown, J. Pommersheim, G. Frohnsdorff. A kinetic model for the hydration of tricalcium silicate. Cement and Concrete Research 15(1):3541, 1985.

[2] E. Gallucci, K. Scrivener. Crystallisation of calcium hydroxide in early age model and ordinary cementitious systems. Cement and Concrete Research 37(4)492-501, 2007.

[3] S. Garrault-Gauffinet, A. Nonat. Experimental investigation of calcium silicate hydrate (C-S-H) nucleation. Journal of Crystal Growth 200(34):565-574, 1999

[4] M. Gartner et al. Chap. 3 of Structure and Performance of Cements (eds. J. Bensted, P. Barnes). Spon Press, 2001.
[5] Š. Hošková, P. Tichá, P. Demo. Determination of $\mathrm{Ca}^{2+}$ ions at early stage of hydrating cement paste. Ceramics-Silikáty 53(2)76-80, 2009.

[6] H. Jennings, P. Pratt. An experimental argument for the existence of a protective membrane surrounding portland cement during the induction period. Cement and Concrete Research 9(4):501-506, 1979.

[7] I. Kanne-Dannetschek, D. Stauffer. Quantitative theory for time lag in nucleation. Journal of Aerosol Science 12(2):105-108, 1981.

[8] S. Preece, J. Billingham, A.King. On the initial stages of cement hydration. Journal of Engineering Mathematics 40:43-58, 2001.

[9] H. Taylor. Cement Chemistry. 2nd edition, Thomas Telford, 2004.

[10] J. Thomas, H. Jennings, J. Chen. Influence of nucleation seeding on the hydration mechanisms of tricalcium silicate and cement. Journal of Physical Chemistry C 113:4327-4334, 2009.

[11] H. Vehkämäki. Classical nucleation theory in multicomponent systems. Springer, 2006. 